\title{
LED INDUSTRIAL SPATIAL STRUCTURE AND ITS EVOLUTION TREND IN HANGZHOU BAY AREA, CHINA
}

\author{
Haiwei FU \\ International Port and Logistics Research Centre, Ningbo \\ University of Technology, Ningbo, 315211 China; \\ E-mail: richard_0518@hotmail.com
}

\begin{abstract}
The numbers of spatial scale structural dimensions and associated structural dimensions in the light emitting diode (LED) industry in Hangzhou Bay Area, China since 2002 were estimated through the fractal method for the prediction of the future evolution trend of the industry. We found that the spatial structure of the LED industry in that area is of strong monopoly but tends to be generally optimal and stable. The morphology in which the main enterprises concentrate in a linear space contributes to the interactions among the enterprises; future spatial structure of LED industry in Hangzhou Bay Area will be a stable state. Changes in external environments and the interaction between internal industrial factors constitute the basic dynamic mechanism for the formation and evolution of LED industrial spatial structure in the Hangzhou Bay Area.
\end{abstract}

Keywords: Hangzhou Bay Area, LED industry, fractal, R/S analysis, spatial structure

\section{INTRODUCTION}

Owing to the increasing popularity of semiconductor lighting, semiconductor light sources, especially light emitting diodes (LEDs), has attracted interest worldwide [1]. The LED industry is considered as one of the high-tech industries with the greatest development prospect in the $21^{\text {st }}$ century [2]. Faced with this enormous market space, several countries have correspondingly promoted LED industrial development plans and started to occupy the global market [3]. The Hangzhou Bay Area is one of the areas with the most developed economy in China. After years of development, the LED industry in this area has gradually developed self-innovative abilities. In addition, a complete industrial chain has been formed. To date, there are more than 2000 LED enterprises engaged in epitaxial wafer, chip, encapsulation, and application in Hangzhou Bay Area. Thus, Hangzhou Bay Area has become one of the largest LED production and supply bases worldwide.

The spatial evolution of the LED industry is a complicated dynamic process with large time span. Clearly revealing this evolution process is a problem needing urgent solution in the current industrial development. However, discussing about problems, such as spatial structure and evolution trend of LED industry, may party improve the competitiveness of LED industry and eventually drive the development of related industrial clusters and realize industrial transformation and upgrade. Therefore, in this study, we attempt to introduce fractal idea into LED industry research, expecting to completely present the spatial structure of the LED industry in the Hangzhou Bay Area and its evolution features since 2002. This work is also a significant supplementation for the research contents and methods of the LED industry.

\section{LITERATURE REVIEW}

The LED industrial field has always been a great concern among academic circles [4]. With conti- 
nuous development of LED technology, related studies have drawn increasing attention [5]. However, not a few scholars have explored the main factors influencing the spatial evolution of LED industry. Taking Taiwan as an example, Ma et al., used the fuzzy analytical hierarchy process method to evaluate the paths for the effective acquisition of core technologies in the LED industry. Their results showed that Taiwan enterprises acquire new technologies by licensing or realize technological independence by building R\&D alliance and attracting relevant enterprises with commercial potential and potential market shares [6]. Yushan Chen et al. discussed the competitive and cooperative behaviour among LED enterprises through patent analysis. Their study contributed to spatial agglomeration in the industry [7]. Meanwhile, Kaa et al. verified that national institutional environment negatively affects the development of regional LED industries by restricting the progress of industrial standardization [8].

In general, existing literature on the spatial structure of LED industry is quite limited, lacking empirical works based on quantitative method. On this basis, a measurement model for spatial structure and evolution trend was established in this research by using fractal methods, such as the measurement of scale structural dimensions and associated structural dimensions and $\mathrm{R} / \mathrm{S}$ trend analysis method. We conducted an empirical study of the LED industry in an area in Hangzhou Bay, China.

The rest of this study is organized as follows. Section 3 expounds the application of the fractal method in LED industry studies. Section 4 conducts empirical analysis of the spatial structure and evolution trend of the LED industry in Hangzhou Bay Area. Section 5 analyzes the dynamic mechanism of spatial evolution of LED industry in Hangzhou Bay Area. The final section summarizes the entire study and draws related conclusions.

\section{RESEARCH METHOD}

\subsection{Measurement of Scale Structural Dimensions}

The LED output values in the main cities of Hangzhou Bay, China are selected as representative indicators of industrial scale. The LED industrial scales are sorted by size, and the logarithms of the ranks and numerical values of the LED industrial scales are used for drawing a scatter diagram. Its fitting trend is also observed. When a linear regression functional relationship is observed between the two, the rank-size distribution of the LED industrial scales is regarded conforming to Zip's law. The concrete computational formula is as follows:

$$
T(r)=T_{1} \times r^{-q},
$$

where $r$ is the scale ran of LED industry $(r=1,2, \ldots, 10), T(r)$ is the urban LED industrial scale with rank as $r, T_{1}$ is the LED industrial scale of the first-rank city, and $q$ is the Zip $f$ parameter. Logarithms at two sides of formula (1) are taken to obtain the following:

$$
\ln T(r)=\ln T_{1}-q \ln r .
$$

A series of data pairs [ $\ln r, \ln T(r)]$ are solved using formula (2). These data pairs are fitted, and if they present linear regression relationship, then the number $D_{f}$ of scale structural dimensions of urban LED industry is

$$
D_{f}=R^{2} / q,
$$

where $R^{2}$ is the coefficient of determination of the fitted equation. Fractal dimension $D_{f}$ has effective geographic significance and reflects the spatial distribution pattern of LED industrial scale structures. If $D_{f}<1$, then the LED industries present a $\mathrm{Pa}$ reto unbalanced distribution. The monopoly of the first-rank city is strong, and the overall development of the LED industry is not mature enough. If $D_{f}=1$, the ratio of the first-rank city and the smallest scale city is rightly equal to the number of cities. If $D_{f}>1$, LED industrial distribution is under relative balance, the number of cities in intermediate rank is large, and the overall development of LED industry is relatively mature.

\subsection{Measurement of Associated Structural Dimensions}

Associated structural dimensions in LED industrial space can be explained as spatial interaction laws between LED industries, which depict relative distribution state of LED industries in various cities within the area. Considering that the spatial distribution of LED industry is generally two-dimensional, its spatial correlation function can be defined as 


$$
C(k)=\frac{1}{N^{2}} \sum_{i, j=1}^{N} H\left(k-d_{i j}\right),
$$

where $C(k)$ is the spatial correlation function; $N$ is the number of cities meeting certain conditions; $k$ is the step length; $d_{i j}$ is the Euclidean distance from city $i$ to city $j ; H$ is the step Heaviside function.

$$
H\left(k-d_{i j}\right)=\left\{\begin{array}{ll}
1 & k>d_{i j} \\
0 & k \leq d_{i j}
\end{array} .\right.
$$

If the spatial distribution of the LED industry is fractal, then $C(k) \propto k^{D_{g}}$ should be met. Here, $D_{g}$ is mainly the associated structural dimensions, and its actual calculation is similar to the measurement method of scale structural dimensions on the map. Euclidean distances between cities are first calculated for obtaining the Euclidean distance matrix. Then, the $C(k)$ value is calculated, and $k$ value is changed. The scatter diagram concerning a series of $[k, C(k)]$ data pairs is obtained, and the value of associated structural dimensions $D_{g}$ can be obtained through linear fitting of data pairs. Under general circumstances, $0 \leq D_{g} \leq 2$. When $D_{g} \rightarrow 0$, the LED industries inside the system concentrate into one point. When $D_{g} \rightarrow 2$, the LED industries inside the system concentrate on a smooth curve. A large $D_{g}$ value means several balanced LED industries are distributed in various cities within the area and in a geographic space. Meanwhile, a small $D_{g}$ value indicates that small space industries are concentrated in the areas.

\subsection{Rescaled Range (R/S) Trend Analysis Method}

Proposed by Hurst in 1951, the R/S method in fractal theory is an analysis method for time sequence [9]. R/S analysis method can be used for studying fractal features and long-term memory process of time sequences and determining the evolution laws and predicting their future development trend. An analysis process using the R/S analysis method is as follows: for time sequence $\{B(t)\}$, $t=1,2, \ldots, n$, and for any positive integer $\tau \geq 1$, then:

1) The mean sequence is calculated as

$$
\langle\xi\rangle \tau=1 / \tau \sum_{t}^{\tau} B(t)
$$

2) The cumulative deviation is computed as

$$
X(t, \tau)=\sum_{\mu=1}^{t}\{\xi(\mu)-\langle\xi\rangle \tau\}
$$

3) The range is calculated as

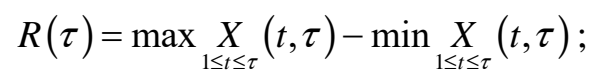

4) The standard deviation is calculated as

$$
S(\tau)=\left[1 / \tau \sum_{t=1}^{\tau}(\xi(t)-\langle\xi\rangle \tau)^{2}\right]^{1 / 2} ;
$$

5) $R / S \equiv R(\tau) / S(\tau)$.

If $R / S \propto \tau^{H}$ is satisfied, then Hurst phenomenon exists in time sequence $\{B(t)\}$ and $H$ is the Hurst index. We determine a whether time sequence trend is persistent or anti-persistent according to the $H$ values. If $H=0.5$, then time sequence is a random walk sequence, and if $0.5<H$ $\leq 1$, then the time sequence evolution is persistent. A large $H$ value means strong persistence. If $0 \leq H$ $<0.5$, then the time sequence evolution is anti-persistent, whereas a small $H$ value indicates strong anti-persistence.

\subsection{Data Source}

We select the LED industries in the 10 main cities in the Hangzhou Bay Area, including not only central cities with high concentration of LED enterprises, such as Shanghai, but also medium and small supporting cities, such as Jiaxing and Shaoxing. These industries cover epitaxial wafer, chip, encapsulation, and application. Sample selection was of certain cover degree and representativeness. Indicator data came from investigations on related guilds, official websites of enterprises, and competent departments.

\section{EMPIRICAL ANALYSIS OF SPATIAL STRUCTURE OF LED INDUSTRY IN HANGZHOU BAY AREA}

\subsection{Analysis of Hierarchical Structure}

The 2017 LED output values of the sample cities were selected as the basic data and sorted according to LED industry size in descending order. 


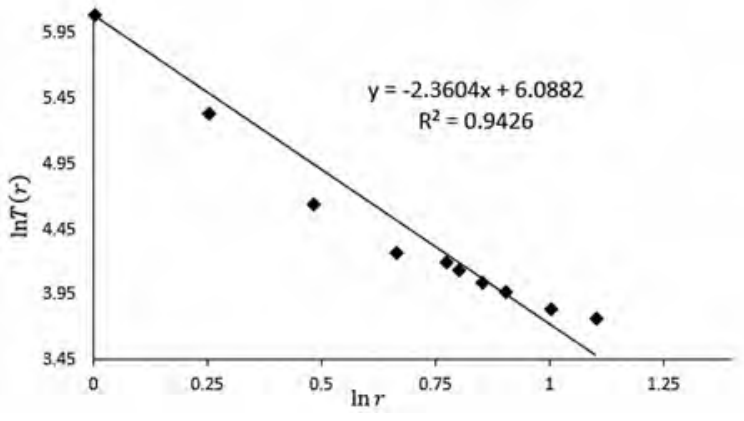

Fig.1. Dial-logarithm diagram of LED industrial rank-size distribution in Hangzhou Bay Area

The purpose was to obtain the rank-size data pairs of the cities. Logarithms of rank data and size data were used for drawing a 2D scatter diagram for fitting. Goodness of fit was used for the evaluation of the existence of fractal features. Fig. 1 presents the rank-size distribution status of LED industries in various cities within Hangzhou Bay Area. In this figure, most scatter points were fitted on the same straight line, coefficient of determination was $R^{2}=0.943$, and obvious and broad scale-free interval was observed with obvious fractal features. The number of dimensions $D_{f}=0.866<1$ was solved according to formulas (2) and (3). The result indicated that LED industries with different hierarchical scales were under a disperse distribution, and the monopoly of first-rank cities was strong. In a similar way, the scale structures of LED industries in Hangzhou Bay Area since 2002 were analyzed, and $D_{f}$ values over the years were obtained. Notably, coefficients $R^{2}$ of determinations of all years reached above 0.9 , suggesting that the spatial scale structures of all the LED industries within the Hangzhou Bay Area have obvious fractal features.

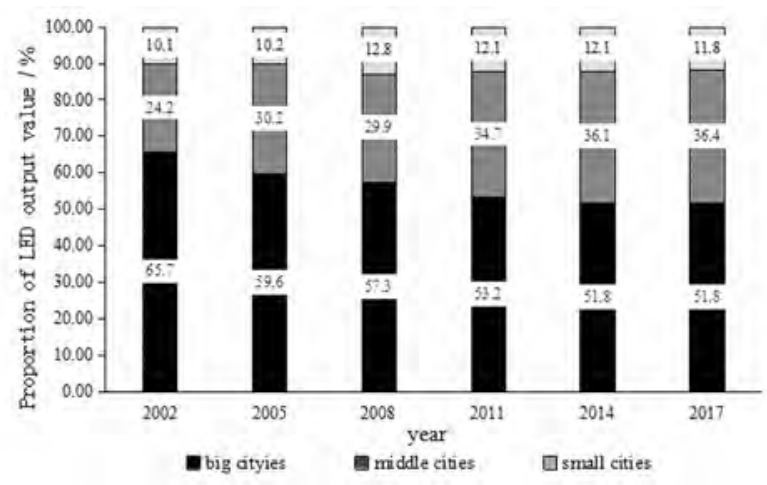

Fig.2. Distributional proportions of LED industrial scales in large, medium, and small cities

As shown in Table 1 , the $D_{f}$ values in all years were smaller than 1 , indicating that the overall development degree of the LED industries in the Hangzhou Bay Area was not mature enough. The LED industries had a non-uniform distribution in different cities, and the first-rank cities had strong monopoly. From the time sequence, the $D_{f}$ values initially presented an increasing evolution trend before levelling off. The $D_{f}$ values from 2002 to 2010 increased obviously, showing that the balance degree of LED industries in space within Hangzhou Bay Area gradually increased. This spatial structural evolution trend promoted industrial development in medium and small cities and facilitated the functional coordination in the entire LED industry. Since 2011, $D_{f}$ values have been basically stabilized at 0.86 . This result, on the one hand, suggests the oversaturation state of investment and construction of medium and small cities and gradual increase of LED industrial scales in various cities. On the other hand, this outcome indicates the "marginal challenge" effect in LED industrial space within the Hangzhou Bay Area.

Table 1. $D_{f}$ Values of LED Industries in Hangzhou Bay Area since 2002

\begin{tabular}{|c|c|c|c|c|c|}
\hline Year & $\boldsymbol{R}^{2}$ & $\boldsymbol{D}_{\boldsymbol{f}}$ & Year & $\boldsymbol{R}^{\mathbf{2}}$ & $\boldsymbol{D}_{\boldsymbol{f}}$ \\
\hline 2002 & 0.946 & 0.642 & 2010 & 0.926 & 0.823 \\
\hline 2003 & 0.942 & 0.683 & 2011 & 0.923 & 0.845 \\
\hline 2004 & 0.938 & 0.737 & 2012 & 0.922 & 0.862 \\
\hline 2005 & 0.934 & 0.746 & 2013 & 0.938 & 0.865 \\
\hline 2006 & 0.931 & 0.732 & 2014 & 0.918 & 0.866 \\
\hline 2007 & 0.928 & 0.763 & 2015 & 0.923 & 0.869 \\
\hline 2008 & 0.925 & 0.752 & 2016 & 0.935 & 0.868 \\
\hline 2009 & 0.934 & 0.778 & 2017 & 0.928 & 0.866 \\
\hline
\end{tabular}


Table 2. Associated structural dimensions of LED industries in Hangzhou Bay Area

\begin{tabular}{|c|c|c|c|c|c|}
\hline $\mathbf{k} / \mathbf{k m}$ & $\boldsymbol{C ( k )}$ & $\mathbf{k} / \mathbf{k m}$ & $\boldsymbol{C ( k )}$ & $\mathbf{k} / \mathbf{k m}$ & $\boldsymbol{C}(\boldsymbol{k})$ \\
\hline 30 & 0.121 & 180 & 0.348 & 330 & 0.835 \\
\hline 60 & 0.162 & 210 & 0.467 & 360 & 0.866 \\
\hline 90 & 0.198 & 240 & 0.584 & 390 & 0.913 \\
\hline 120 & 0.247 & 270 & 0.676 & 420 & 0.952 \\
\hline 150 & 0.289 & 300 & 0.789 & 450 & 1 \\
\hline
\end{tabular}

For the further analysis of spatial pattern evolution of LED industries in Hangzhou Bay Area, the main cities were divided into large, medium, and small types. The distributional proportions of LED industrial scales among the three-type cities are illustrated in Fig. 2.

Overall, the scale structures of LED industries in the Hangzhou Bay Area had obvious monopoly features over the years. In the scale ranking, Shanghai occupies approximately $50 \%$ of the entire LED industrial scale. From time sequence evolution, LED industries gradually migrated from large cities to medium and small cities. The LED output value increased obviously especially in small cities, and the spatial structure of the entire LED industry tended to be optimal and stable. In 2002-2011, the proportion of the LED output values in large cities remarkably decreased, whereas those of medium and small cities increased obviously with continuously optimized spatial structure. After 2011, change trends of proportions of LED output values in various types of cities were becoming small, and the proportion of LED output values in small cities even slightly decreased. The spatial structure of the entire LED industry in Hangzhou Bay Area also tended to be stable.

\subsection{Analysis of Associated Structure}

For the construction of a $10 \times 10$ matrix, Euclidean distances between every two industrial clusters (straight-line distance) in the sample cities were measured by using a geographic information system platform. Step length $k=30 \mathrm{~km}$ was used as a scale, and a series of $C(k)$ values were obtained by changing $k$ value, as shown in Table 2. On this basis, the number of dimensions and coefficient of determination of associated structure were obtained as $D_{g}=1.143$ and $R^{2}=0.968$ with favourable goodness of fit. The number of dimensions of associated structure was approximately 1 . This value indicated that LED industries in the cities were concentrated within a single linear spatial range with strong spatial correlation. This finding was in accordance with the realistic situation. This linear space was largely a supply chain channel constructed by connecting "Shanghai-Hangzhou" Huhang line and "Hangzhou-Ningbo" Hangyong line but also indicated that LED industries in inland cities were not developed enough. Thus, high requirements had been proposed for improvement of inland supply chain network and industrial competitiveness within Hangzhou Bay Area.

\subsection{Research and Judgment of Development Trend}

Year 2002 was taken as the base point, and R/S analysis method was used to analyze time sequence evolution trend of numbers $D_{f}$ of dimensions of LED industries within Hangzhou Bay Area as shown in Table 3. Overall, Hurst index presented a descending trend, indicating continuously weakened persistence of change of $D_{f}$ values in the same direction but with gradually minimized descending amplitude. Before 2013, index was always

Table 3. Hurst indexes of spatial evolution of LED industries within Hangzhou Bay Area

\begin{tabular}{|c|c|c|c|c|}
\hline Period & $\begin{array}{c}\mathbf{2 0 0 2 -} \\
\mathbf{2 0 0 6}\end{array}$ & $\begin{array}{c}\mathbf{2 0 0 2 -} \\
\mathbf{2 0 0 7}\end{array}$ & $\begin{array}{c}\mathbf{2 0 0 2}- \\
\mathbf{2 0 0 8}\end{array}$ & $\begin{array}{c}\mathbf{2 0 0 2}- \\
\mathbf{2 0 0 9}\end{array}$ \\
\hline H & 0.784 & 0.649 & 0.658 & 0.732 \\
\hline Period & $\begin{array}{c}2002- \\
2010\end{array}$ & $\begin{array}{c}2002- \\
2011\end{array}$ & $\begin{array}{c}2002- \\
2012\end{array}$ & $\begin{array}{c}2002- \\
2013\end{array}$ \\
\hline H & 0.633 & 0.562 & 0.532 & 0.515 \\
\hline Period & $2002-$ & $2002-$ & $2002-$ & $2002-$ \\
2014 & 2015 & 2016 & 2017 \\
\hline H & 0.485 & 0.498 & 0.506 & 0.497 \\
\hline
\end{tabular}


greater than 0.5 , and $D_{f}$ value evolution would present persistent development trend. This result suggested a continuous growth. After 2011, index was approximately 0.5 , and $D_{f}$ value evolution would present stable trend with small fluctuation. In summary, as Hurst index continues to fluctuate at 0.5 , the spatial structure of LED industries in Hangzhou Bay Area would not change greatly within a period in the future.

\section{DYNAMIC MECHANISM OF SPATIAL EVOLUTION OF LED INDUSTRIES IN THE HANGZHOU BAY AREA}

\subsection{External Factors}

The external influence factors of spatial evolution of LED industries are mainly manifested at three aspects. The first aspect is the driving effect of associated industries [10]. LED products are important supporting products of various manufacturing industries. The Hangzhou Bay Area is a developed manufacturing industry, and the development of industries, such as automobile, requires a large quantity of LED products. This phenomenon has provided enormous demand market for the LED industry [11]. The second is governmental policy support. Governmental guidance is a decisive factor of the spatial evolution of LED industries [12]. In recent years, the local government of the Hangzhou Bay Area has planned multiple LED industrial parks and industrial bases and attracted many LED enterprises by providing preferential policies to form enormous agglomeration effect. The third is agglomeration of scientific and technological innovation elements [13]. LED industry development cannot go without support from scientific studies. Hangzhou Bay Area is agglomerated with many famous colleges, universities, and scientific research institutes with dense intelligent elements. Domestic top-end LED R \& D centre has also been formed. R \& D and patent service can provide guarantee for spatial agglomeration and development of LED industries in this area.

\subsection{Internal Factors}

Internal influence factors of spatial evolution of LED industries mainly include three aspects. The first is competition and cooperation between enterprises. Internal competition between enterprises is the direct cause for "marginal challenge" [14]. With the improvement and elevation of their own environments, medium and small cities have adopted a series of strategies, such as price competition, policy support, and fiscal subsidies to attract LED enterprises to set up production and R \& D bases to dilute industrial resources of large cities and narrow regional gaps. Meanwhile, industrial-chain cooperation is formed between cities to promote joint growth of LED industrial scales in these cities, and this growth usually presents high synchronization and promotes stability of spatial structure of LED industries. The second is core technological control. LED industries have strong technological orientation. Enterprises with strong R \& D abilities within the industry usually control core technologies and patents in this industry. These enterprises can become core enterprises on LED industrial chain and can constitute and develop the entire industrial chain to decide the spatial layout of the regional industry. The third is industrial talents. LED industry involves multiple disciplines, such as light, electricity, and heat. Thus, a large number of comprehensive professional and technical talents are needed. Nowadays, shortage of high-end talents has become the key bottleneck restricting LED industrial development in Hangzhou Bay Area. The cities that can provide good development environment for talents can attract related technical talents and attract agglomeration of high-end enterprises in the industrial chain to form an industrial development highland.

\section{CONCLUSIONS}

LED industry in Hangzhou Bay Area was taken as an example in this research, and fractal method was used to analyze the spatial structure and evolution trend of LED industry. The research shows the following: (1) spatial structure of LED industry in Hangzhou By area is not only of monopoly feature but also of "marginal challenge" spatial effect and generally tends to be optimal and stable; (2) LED industries in Hangzhou Bay Area are distributed within a linear range with strong interaction between main cities; (3) persistence of spatial structural fluctuation of LED industries in Hangzhou Bay Area decreases and finally tends to be stable; and (4) the abovementioned dynamic mechanism of spatial structure and its evolution is not only related to peripheral economic environment and poli- 
cy environment of LED industry but also is closely related to internal development factors within LED industry.

\section{ACKNOWLEDGEMENT:}

The authors are grateful for the support provided by the National Natural Science Foundation of China (No. 41501142).

\section{REFERENCES:}

1. Ciriminna, R., Albanese, L., Meneguzzo, F., Pagliaro, M. LED street lighting: a looking ahead perspective. Green, 2015. V5, \#1-6, pp.83-94.

2. Gayral, B. LEDs for lighting: Basic physics and prospects for energy savings. Comptes Rendus Physique, 2017. V18, \#7-8, pp.453-461.

3. Khorasanizadeh, H., Parkkinen, J., Parthiban, R., Moore, J D. Energy and economic benefits of LED adoption in Malaysia. Renewable \& Sustainable Energy Reviews, 2015. V49, pp.629-637.

4. Duee, D. LED lighting industry: Opportunity or health hazard? Light \& Engineering, 2012. V20, \#3, pp.23-24.

5. Rammohan, A., Kumar Ramesh, C. A review on effect of thermal factors on performance of high power light emitting diode (HPLED). Journal of Engineering Science and Technology Review, 2016. V9, \#4, pp.165-176.

6. Ma, D., Hung, S W. An Integrated Framework for the Selection and Acquisition of Core Technologies: The
Case of Taiwan's LED Industry. Long Range Planning, 2015. V48, \#6, pp.381-397.

7. Yushan, C., Biyu, C. Utilizing patent analysis to explore the cooperative competition relationship of the two LED companies: Nichia and Osram. Technological Forecasting and Social Change, 2011. V78, \#2, pp.294-302.

8. Kaa, G V D., Greeven, M. LED standardization in China and South East Asia: Stakeholders, infrastructure and institutional regimes. Renewable and Sustainable Energy Reviews, 2017. V72, pp.863-870.

9. Mason, D M. The Hurst phenomenon and the rescaled range statistic. Stochastic Processes and their Applications, 2016. V126, \#12, pp.3790-3807.

10. Diez, O., Larrode-Pellicer, E., Muerza-Marin, $\mathrm{V}$. Improving competitiveness in companies of the automotive supply industry: Twin-equipment strategy. DYNA, 2017. V92, \#4, pp.380-381.

11. Jing, W., Yixi, C., Xiaohua, L., Yunfei, S., Yachao, B., Jun, W., Yunxi S. Experimental study on optical-thermal associated characteristics of LED car lamps under the action of ionic wind. Microelectronics Reliability, 2018. V82, pp.113-123.

12. Wei, W. The role of the government in the development of the LED industry. Light \& Engineering, 2016. V24, \#3, pp.128-131.

13. Jurene, S., Jureniene, V. Creative cities and clusters. Transformations in Business \& Economics, 2017. V16, \#2, pp.214-234.

14. Ikeda, K., Akamatsu, T., Kono, T. Spatial period-doubling agglomeration of a core-periphery model with a system of cities. Journal of Economic Dynamics and Control, 2012. V36, \#5, pp.754-778.

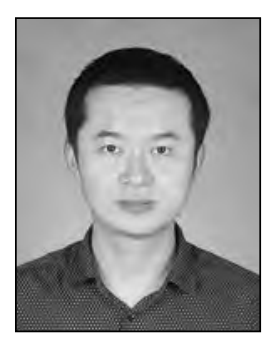

\section{Haiwei Fu,}

Doctor of Engineering, Associate Professor. Graduated from the Shanghai Maritime University. Fellow of the Ningbo University of Technology of China 